\title{
A detailed semiologic analysis of childhood psychogenic nonepileptic seizures
}

\author{
*†Léna Szabó, *Zsuzsanna Siegler, łLászló Zubek, §Zoltán Liptai, $₫$ Ivett Körhegyi, \\ $\dagger$ †Boglárka Bánsági, and *András Fogarasi
}

\begin{abstract}
*Department of Neurology, Bethesda Children's Hospital, Budapest, Hungary; $\nmid 2 n d$ Department of Pediatrics, Semmelweis University, Budapest, Hungary; $\ddagger$ Department of Anesthesiology and Intensive Therapy, Semmelweis University, Budapest, Hungary; $\S$ Department of Pediatrics, Szent László Hospital, Budapest, Hungary; and ๆDepartment of Pediatrics, Debrecen University, Debrecen, Hungary
\end{abstract}

\section{SUMMARY}

Purpose: Psychogenic nonepileptic seizure (PNES) is an important differential diagnostic problem in patients with or without epilepsy. There are many studies that have analyzed PNES in adults; currently, however, there is no systematic assessment of purely childhood PNES semiology. Our study based on a large pediatric video-electroencephalography (EEG) monitoring (VEM) cohort, provides a detailed analysis of childhood PNES and assesses the usability of the current classification system described in adults.

Methods: Medical and video-EEG records of 568 consecutive children (younger than 18 years) who underwent videoEEG monitoring (VEM) at our hospital were reviewed. Aura, type of movement, anatomic distribution, synchrony, symmetry, eye movement, responsiveness, vocalization, hyperventilation, vegetative and emotional signs, presence of eyewitness, and duration of the event were recorded among children with the diagnosis of PNES. We also compared our data with those of earlier adult studies.

Key Findings: Seventy-five archived PNES of 27 children (2I girls; age 8- 8 years) were reanalyzed. Nine children (33\%) had the diagnosis of epilepsy currently or in the past. Mean age at the time of PNES onset was I I.6 (standard deviation 3.2) years. Mean duration of PNES was longer (269 s) compared to seizures of the epileptic group (83 s; $p=0.002)$. Eyewitnesses (mostly parents) were present in $\mathbf{8 9} \%$ of cases. Eighty percent of PNES had an abrupt start, with $68 \%$ also ending abruptly. In only I $5 \%$ of events were the patients eyes closed at the beginning of the attack.
Patients were unresponsive in $34 \%$. The most frequent motor sign was tremor (25\%) with the upper, rather than lower limbs more frequently involved. Pelvic thrusting was seen in only two attacks. Emotional - mostly negative - signs were observed during 32 PNES (43\%). Based on Seneviratne et al.'s classification, 18 events (24\%) were classified as rhythmic motor PNES, only half the frequency of that previously described in adults. No hypermotor PNES was found. The frequency of complex motor PNES (13\%) and mixed PNES (4\%) showed similar frequency in children as in adults. Dialeptic PNES was found more frequently among younger children. All PNES belonged to the same semiologic type in 23 patients $(85 \%)$. Significance: Because homogeneity of PNES within a patient was high in the pediatric population, we found it useful to classify PNES into different semiologic categories. Dialeptic PNES seems to be more frequent among younger children. Tremor is the most frequent motor sign and usually accompanied by preserved responsiveness in childhood. Negative emotion is commonly seen in pediatric PNES, but pelvic thrusting is a rare phenomenon. We, therefore, suggest a modification of the present classification system in which PNES with motor activity is divided into minor and major motor PNES, and the latter group is subdivided into synchron rhythmic motor and asynchron motor PNES. We believe that our study, a detailed analysis on the semiology and classification of purely childhood PNES might assist the early and precise diagnosis of nonepileptic paroxysmal events.

KEY WORDS: Psychogenic nonepileptic seizures, Seizure semiology, Classification, Children, Video-EEG.
Psychogenic nonepileptic seizure (PNES) is an observable abrupt paroxysmal change in behavior or consciousness that resembles an epileptic seizure, but is not accompanied 2012.

Accepted December 29, 2011; Early View publication February 14,

Address correspondence to Léna Szabó, 7-9 Tüzoltó Street, Budapest 1094, Hungary. E-mail: szabo.lena@gmail.com

Wiley Periodicals, Inc.

(C) 2012 International League Against Epilepsy by the electrophysiologic changes or clinical evidence for epilepsy. There are no somatic causes for the seizures, but a strong suspicion or positive evidence for a psychogenic factor (Bodde et al., 2009). The prevalence of PNES has been estimated at 2-33/100,000 (Benbadis \& Allen Hauser, 2000); however, up to $20 \%$ of patients seen at epilepsy referral centers have psychogenic seizures (Lesser, 1996). The onset of PNES is typically in the second or third decade of life (Reuber, 2008). In a group of patients 
diagnosed with PNES, most patients experienced the first episode between 10 and 19 years; however, PNES was diagnosed only between 20 and 40 years (Reuber et al., 2002; Reuber, 2008). Several studies described various semiologic features of PNES (Gulick et al., 1982; Gates et al., 1985; Meierkord et al., 1991; Leis et al., 1992; Lancman et al., 1993). Despite the well-known benefits of a good classification system in the recognition and management of PNES, only a few studies have tried to semiologically classify psychogenic seizures (Groppel et al., 2000; An et al., 2010; Seneviratne et al., 2010; Hubsch et al., 2011) and only one article assessed semiology of a purely childhood PNES group (Patel et al., 2007). The current study based on a large pediatric video-electroencephalography (EEG) monitoring (VEM) database, provides a detailed analysis of childhood PNES and assesses the usability of the current classification system described in adults (Seneviratne et al., 2010).

\section{Methods}

We retrospectively reviewed medical and video-EEG records of all children who underwent VEM in our hospital between June 2001 and June 2011. Those diagnosed with PNES, with or without epilepsy, were selected for this study. The diagnosis was made based on clinical data and video-EEG monitoring by at least two epilepsy specialists unaware of the goal of this study (all diagnoses were completed before the start of this retrospective analysis). The following criteria were used to diagnose PNES (Seneviratne et al., 2010): (1) at least one typical event was captured on video-EEG, (2) the EEG did not show any electrographic ictal rhythm during the event, (3) no postictal slowing was seen on the EEG, and (4) there was no evidence of an alternative neurologic diagnosis for this event. Children with recognized paroxysmal disorders such as parasomnias or extrapyramidal movement disorders were excluded.

All patients had continuous inpatient VEM for an average period of 3 days (1-5 days). Scalp electrodes were placed in accordance with the 10-20 international electrode system. Patients' consciousness and reactivity were tested during and after the attacks by EEG assistants. EEG and audio visual signals were acquired and analyzed using Brain Quick (Micromed S.p.A, Mogliano Veneto (TV), Italy) video-EEG system and System Plus software (Micromed S.p.A.).

The semiology of each clinical event was visually analyzed in detail (L.S. and A.F.) and entered into a statistical database (Statistica, StatSoft Inc., Tulsa, OK, U.S.A.). Semiologic assessment of events were based on a previously described detailed seizure analysis (Fogarasi et al., 2007b): type and anatomic distribution of the movement (extremities, head, trunk, pelvis), synchrony, symmetry, eye movement, responsiveness, vocalization, hyperventilation, vegetative signs, presence of emotional signs and aura, onset (abrupt or gradual), course, presence of eyewitness, and duration of the event were recorded and tabulated. We used the results of Seneviratne et al. (2010) to classify events into distinct groups: 1. Rhythmic Motor PNES, 2. Hypermotor PNES, 3. Complex motor PNES, 4. Dialeptic PNES, 5. Nonepileptic Aura, and 6. Mixed PNES.

Statistical analysis was made by STATISTICA (version 9.1.; StatSoft Inc., 2010, http://www.statsoft.com). Means, standard deviation, median, minimum, and maximum for continuous variables, and frequency for categorical variables were calculated. Nonparametric tests (Mann-Whitney $U$ test or Kruskal-Wallis test) were used to compare groups.

Written informed consent was obtained from each patient or the patient's parent(s) before VEM. Our study was authorized by the ethical committee of the Bethesda Children's Hospital.

\section{ResUlts}

\section{Patients}

In this period, 568 children underwent VEM in our department with the clinical question of either differential diagnosis of epilepsy, better epilepsy syndrome classification, or presurgical evaluation. Twenty-seven of them (4.8\%) were diagnosed with PNES. A total of 275 (1-100/ child, median: 3/child) PNES were recorded. From the 27 patients, $21(78 \%)$ were girls, the age of children at VEM was $8-18$ (mean $14.8 \pm 2.8$ ) years. If a child had several seizures with the same or very similar semiology, usually 6-8 (clinically most relevant) events were archived. Finally 75 (1-8, median 2/child) archived events of the 27 children were available for detailed analysis. When we compared the age of the children in the PNES group to all other patients who underwent VEM (7.9 \pm 5.1 years, 235 girls $)$, children with PNES were older $(z=-6.21, \mathrm{p}<0.001)$ (Fig. 1). In nine children $(33.3 \%)$ PNES was accompanied with an epilepsy diagnosis at the same time or in the past. The mean age at the time of PNES onset was $11.6 \pm 3.2$ years.

\section{Events}

The mean duration of PNES was $269 \pm 549$ (range $1-3,417)$ seconds compared to seizures in the epileptic group $83.2 \pm 222.4 \mathrm{~s}(z=-3.72, \mathrm{p}=0.002)$.

At the start of PNES episodes, there was an eyewitness (mostly parents) in the VEM room in $89 \%$ of cases. Eighty percent of events had an abrupt start, with $68 \%$ also ending abruptly. In $15 \%$ of events, the patients had their eyes closed at the beginning and $22 \%$ had their eyes closed during the whole attack. Patients were unresponsive during $34 \%$ of all events.

The most frequent motor sign was tremor (25\%) involving the upper limbs more frequently than the lower. Pelvic thrusting was seen only in two attacks. 


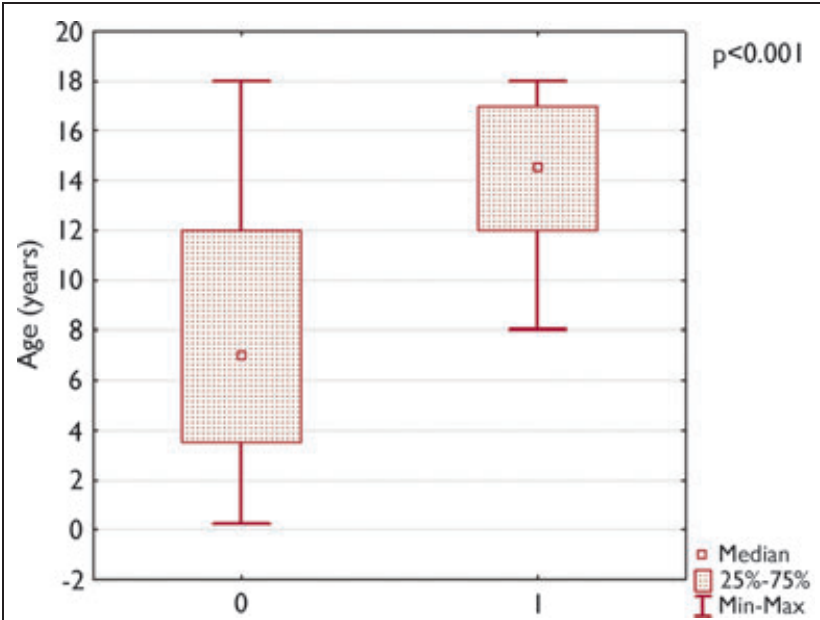

Figure I.

Mean age of children with PNES and children with epilepsy who underwent VEM. Children with diagnosis of PNES and epilepsy together are in PNES group. 0, children with epilepsy; I, children with PNES.

Epilepsia (C) ILAE

Emotional signs were observed during 32 attacks (43\%). They were negative in almost all children, except two cases when laughing or smiling could be observed (Table 1).

Based on Seneviratne et al.'s classification in our cohort, 18 events (24\%) were classified as rhythmic motor PNES, only half the frequency of that previously described in adults (Seneviratne et al., 2010). No hypermotor PNES was found. The frequency of complex motor PNES (13\%) and mixed PNES (4\%) showed similar frequency in children as in adults. Dialeptic PNES was found more frequently in children $(29 \%)$ then in adults $(11 \%)$ (Table 2).

Within the patients, we also studied the homogeneity of PNES types. In 23 patients ( $85 \%$ ) all PNES belonged to the same semiologic type. Comparing the different types of childhood PNES, the youngest children (mean age 13.4 years) belonged to the dialeptic PNES group.

\section{Discussion}

\section{Frequency}

The prevalence of PNES among children who underwent VEM was $4.8 \%$, which is similar to the $1.8-7.8 \%$ from earlier adult studies (Bye et al., 2000; Kotagal et al., 2002; Patel et al., 2007; Kutluay et al., 2010). Seventy-eight percent of our patients were female. This female predominance is seen in adults (67-74\%) (Lesser, 1996; Groppel et al., 2000; Seneviratne et al., 2010; Hubsch et al., 2011) as well as in children (74\%) (Lancman et al., 1994), although some authors have described a decrease in this tendency in younger groups (Kramer et al., 1995; Kotagal et al., 2002; Patel et al., 2007). Previous studies reported coexisting epilepsy in $15-72 \%$ of children with PNES (Holmes et al., 1980; Kramer et al., 1995; Irwin et al.,
2000; Kotagal et al., 2002; Patel et al., 2007), our ratio of $33 \%$ is also within this wide range.

\section{Length}

PNES typically lasts longer than epileptic seizures (Gates et al., 1985; Reuber, 2008); this could also be observed in our pediatric population. Although in adult PNES the gradual onset is the typical form (Holmes et al., 1980; Meierkord et al., 1991; Lancman et al., 1994), in our cohort, $80 \%$ of the episodes started abruptly, which could make the differential diagnosis from epilepsy more difficult.

\section{Emotion}

Weeping is a relatively common and specific clinical feature of pseudoseizures (Bergen \& Ristanovic, 1993). Walczak \& Bogolioubov (1996) found nonverbal communication (weeping, whimpering, or crying) in $14 \%$ of PNES. Nonverbal communication could be observed in $16 \%$ of our archived events (mostly in complex motor and mixed groups), whereas verbal communication was present in 32\%. Emotional signs (weeping, crying, painful facial expression, fear, or laughing) were relatively high (43\%) during the archived attacks and mostly expressed negative emotions. However, this high ratio does not help differentiation from epileptic seizures. Of interest, our earlier VEM study demonstrated that negative emotions also dominate childhood epileptic seizures (Fogarasi et al., 2007a).

\section{Motor signs}

Tremor is typically seen in PNES (Groppel et al., 2000), although some authors found dystonic movement even more frequent (Hubsch et al., 2011). In our group, tremor was the most frequent ictal motor sign, appearing in $25 \%$ of all seizures. Pelvic thrusting is rarely seen in children, whereas it is frequent in PNES and also in frontal lobe epilepsy in adults (Geyer et al., 2000). Considering the entire group, upper limbs were more often involved than lower limbs. This was typical in the rhythmic motor group, whereas in the complex motor group, similarly to adults, lower limb involvement was more common, (Groppel et al., 2000; Seneviratne et al., 2010).

\section{Semiologic classification}

The need for PNES classification was referred to earlier. The most frequently used classification system is based on dominant symptoms and differentiates attacks with loss of consciousness (33\%), and attacks with prominent motor activity (66\%) (Meierkord et al., 1991). Some authors divided PNES according to laterality of symptoms and described four major ictal patterns: bilateral motor episodes (15 of 27); unilateral motor episodes (3 of 27); multiple behavioral phenomena ( 8 of 27); impaired response but no observable behavior (3 of 27) (Gulick et al., 1982). Also described were catatonic/thrashing/subjective spells/ 


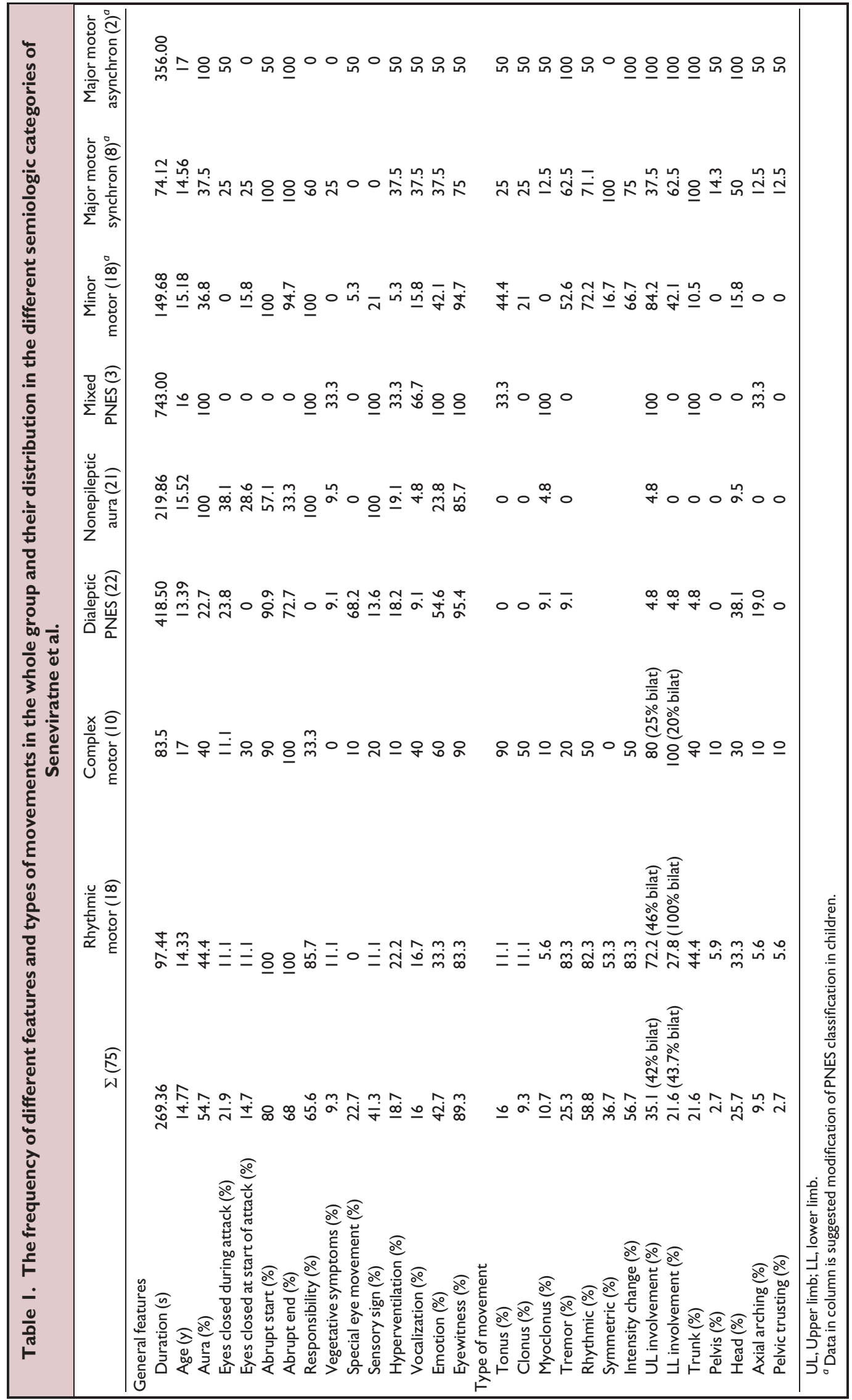




\begin{tabular}{|c|c|c|c|}
\hline Type of attack & $N(75)$ & Percentage & $\begin{array}{l}\text { Percentage } \\
\text { (Seneviratne } \\
\text { et al., 2010) }\end{array}$ \\
\hline Rhythmic tremor or trembling & 18 & 24 & 46.7 \\
\hline Hyperkinetic hypermotor & & 0 & 3.3 \\
\hline Complex motor & 10 & 13.3 & 10 \\
\hline Dialeptic & 22 & 29.3 & 11.2 \\
\hline Nonepileptic aura & 21 & 28 & 23.6 \\
\hline Mixed PNES & 3 & 4 & 5.2 \\
\hline Minor motor ${ }^{a}$ & 19 & 25.3 & \\
\hline Major motor ${ }^{a}$ & 10 & 13.3 & \\
\hline Dialeptic $^{a}$ & 22 & 29.3 & \\
\hline Nonepileptic aura $^{a}$ & 21 & 28 & \\
\hline Mixed PNES ${ }^{a}$ & 3 & 4 & \\
\hline
\end{tabular}

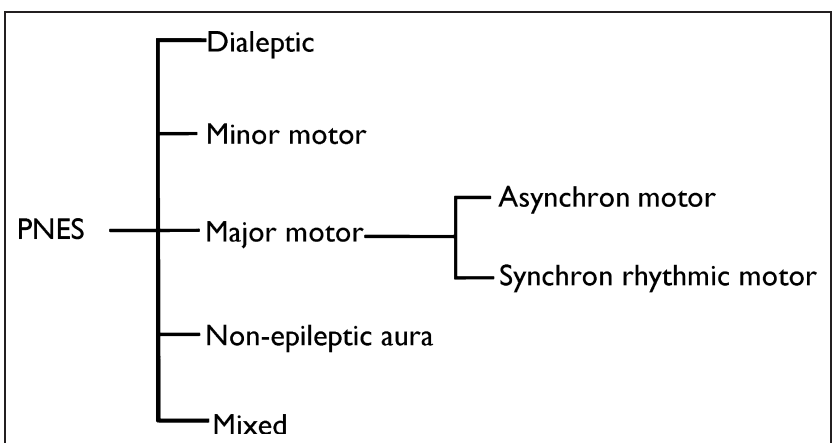

Figure 2.

Schema of the suggested modification on the classification system for children with PNES.

Epilepsia (c) ILAE

tremors/automatisms/intermittent behaviors as groups of PNES (Selwa et al., 2000). In childhood based on the extent of motor activity, prominent motor, subtle motor, and a group containing both types of activity were described by Patel et al. (2007). They found that in children younger than 13 years, nonepileptic seizures commonly manifested as subtle motor activity, whereas in the adolescent group, prominent motor activity was more frequent (Patel et al., 2007). Kramer et al. (1995) found staring and unresponsiveness as more common manifestations in children (69 years), with mainly motor activity in adolescents (10-17 years), whereas Kotagal et al. (2002) did not find significant differences in the clinical semiology of nonepileptic seizures between these two age groups. However, this study examined all types of paroxysmal nonepileptic events including parasomnias, stereotypic movement, movement disorders, and hypnic jerks that are accompanied by motor activities and more common in younger children.

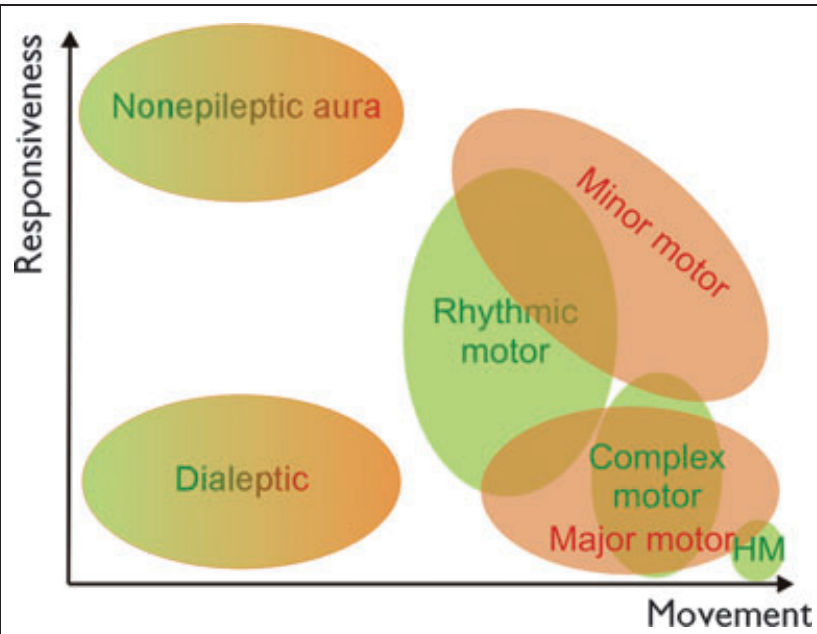

Figure 3.

The different categories of PNES are located in a two-dimensional space, where the $x$-axis is the quantity and complexity of movements, whereas along the $y$-axis, responsiveness is increasing. Green indicates the categories invented by Seneviratne et al., with light red showing our suggestions. As seen in the figure, the minor motor category contains elements of rhythmic motor and complex motor aggregation and locates clearly higher in the $y$-axis as the major motor group. HM, hypermotor

Epilepsia C ILAE

Our data also support an earlier finding that a sedentary, dialeptic form of PNES could be a feature of early childhood (under 14 years of age), since dialeptic PNES was twice as frequent in our pediatric population as in adults (Seneviratne et al., 2010). Moreover, in our study population the mean age is the lowest in the dialeptic group. Of interest, it runs parallel with the observation of earlier epilepsy studies, where the complexity of seizures increases with age (Fogarasi et al., 2006, 2007b).

Although psychosomatic symptoms change frequently within a patient, the semiology of PNES attacks is usually stable in both the pediatric and adult population (Patel et al., 2007; Seneviratne et al., 2010). In children, the length of attacks, similarly to adults, was longest in the dialeptic group.

\section{Proposal of some modification on PNES classification in children}

We have used auditory or visual stimuli and simple command to assess responsiveness during PNES attacks. Based on our experiences, tremor is the most frequent motor symptom in childhood PNES; this is not usually accompanied by unconsciousness (responsiveness was maintained in 86\%). Therefore, we do not feel it fully appropriate to classify these attacks as rhythmic motor seizure as described by Seneviratne et al. (their responsiveness ratio was only $16 \%)$. After reviewing several childhood PNES attacks, we believe that an event with tremor of one extremity and a 
grand mal-like tonic-clonic attack affecting four extremities should not be put into the same category. Based on the attacks observed in children, we subcategorized the mainly motor PNES to minor motor and major motor seizures. The minor motor seizure is characterized by a homogenous motor activity (mainly tremor but also tonic like movement could be observed), which is localized, mainly synchronized, and more often involves the upper limb accompanied by retained responsiveness. Seizures with more complex movements involving several limbs or migrating and appearing as various types, are classified as major motor seizures. Disturbed consciousness is often seen in this group. The major motor group could be subdivided based on movement synchrony as asynchron motor and synchron rhythmic motor groups (Figs. 2 and 3).

\section{Conclusion}

Because homogeneity of PNES within a patient was also high in the pediatric population, we found it useful to classify PNES into different semiologic categories. Dialeptic PNES seems to be more frequent among children, mainly in younger age groups. Tremor is the most frequent motor sign and is usually accompanied by preserved responsiveness in childhood. Negative emotion is commonly seen in pediatric PNES, but pelvic thrusting is a rare phenomenon. We, therefore, suggest some modification on the present classification system in which PNES with motor activity is divided into minor and major motor PNES and major motor PNES is subdivided into synchron rhythmic motor and asynchron motor PNES.

The study has some limitation due to the retrospective study design. We have analyzed only the records of archived attacks and it could result in some data loss or bias despite the most accurate choosing of clinically relevant events during the archiving procedure. Another limitation of our study is the relatively low number of cases. However, it is difficult to gather large childhood cohorts of PNES; therefore, multicenter studies with higher numbers of patients are necessary to confirm our data in the future.

\section{ACKnowledgments}

AF was supported by the Bolyai Scholarship of the Hungarian Academy of Sciences.

\section{Disclosure}

None of the authors has any conflict of interest to disclose. We confirm that we have read the Journal's position on issues involved in ethical publication and affirm that this report is consistent with those guidelines.

\section{REFERENCES}

An DM, Wu XT, Yan B, Mu J, Zhou D. (2010) Clinical features of psychogenic nonepileptic seizures: a study of 64 cases in southwest China. Epilepsy Behav 17:408-411.
Benbadis SR, Allen Hauser W. (2000) An estimate of the prevalence of psychogenic non-epileptic seizures. Seizure 9:280-281.

Bergen D, Ristanovic R. (1993) Weeping as a common element of pseudoseizures. Arch Neurol 50:1059-1060.

Bodde NM, Brooks JL, Baker GA, Boon PA, Hendriksen JG, Mulder OG, Aldenkamp AP. (2009) Psychogenic non-epileptic seizures - definition, etiology, treatment and prognostic issues: a critical review. Seizure 18:543-553.

Bye AM, Kok DJ, Ferenschild FT, Vles JS. (2000) Paroxysmal non-epileptic events in children: a retrospective study over a period of 10 years. J Paediatr Child Health 36:244-248.

Fogarasi A, Janszky J, Tuxhorn I. (2006) Peri-ictal lateralizing signs in children: blinded multiobserver study of 100 children $<$ or $=12$ years. Neurology $66: 271-274$.

Fogarasi A, Janszky J, Tuxhorn I. (2007a) Ictal emotional expressions of children with partial epilepsy. Epilepsia 48:120-123.

Fogarasi A, Tuxhorn I, Janszky J, Janszky I, Rasonyi G, Kelemen A, Halasz P. (2007b) Age-dependent seizure semiology in temporal lobe epilepsy. Epilepsia 48:1697-1702.

Gates JR, Ramani V, Whalen S, Loewenson R. (1985) Ictal characteristics of pseudoseizures. Arch Neurol 42:1183-1187.

Geyer JD, Payne TA, Drury I. (2000) The value of pelvic thrusting in the diagnosis of seizures and pseudoseizures. Neurology 54:227229.

Groppel G, Kapitany T, Baumgartner C. (2000) Cluster analysis of clinical seizure semiology of psychogenic nonepileptic seizures. Epilepsia 41:610-614.

Gulick TA, Spinks IP, King DW. (1982) Pseudoseizures: ictal phenomena. Neurology 32:24-30.

Holmes GL, Sackellares JC, Mckiernan J, Ragland M, Dreifuss FE. (1980) Evaluation of childhood pseudoseizures using EEG telemetry and video tape monitoring. J Pediatr 97:554-558.

Hubsch C, Baumann C, Hingray C, Gospodaru N, Vignal JP, Vespignani H, Maillard L. (2011) Clinical classification of psychogenic non-epileptic seizures based on video-EEG analysis and automatic clustering. J Neurol Neurosurg Psychiatry 82:955-960.

Irwin K, Edwards M, Robinson R. (2000) Psychogenic non-epileptic seizures: management and prognosis. Arch Dis Child 82:474-478.

Kotagal P, Costa M, Wyllie E, Wolgamuth B. (2002) Paroxysmal nonepileptic events in children and adolescents. Pediatrics 110:e46.

Kramer U, Carmant L, Riviello JJ, Stauffer A, Helmers SL, Mikati MA, Holmes GL. (1995) Psychogenic seizures: video telemetry observations in 27 patients. Pediatr Neurol 12:39-41.

Kutluay E, Selwa L, Minecan D, Edwards J, Beydoun A. (2010) Nonepileptic paroxysmal events in a pediatric population. Epilepsy Behav 17:272-275.

Lancman ME, Brotherton TA, Asconape JJ, Penry JK. (1993) Psychogenic seizures in adults: a longitudinal analysis. Seizure 2:281-286.

Lancman ME, Asconape JJ, Graves S, Gibson PA. (1994) Psychogenic seizures in children: long-term analysis of 43 cases. $J$ Child Neurol 9: 404-407.

Leis AA, Ross MA, Summers AK. (1992) Psychogenic seizures: ictal characteristics and diagnostic pitfalls. Neurology 42:95-99.

Lesser RP. (1996) Psychogenic seizures. Neurology 46:1499-1507.

Meierkord H, Will B, Fish D, Shorvon S. (1991) The clinical features and prognosis of pseudoseizures diagnosed using video-EEG telemetry. Neurology 41:1643-1646.

Patel H, Scott E, Dunn D, Garg B. (2007) Nonepileptic seizures in children. Epilepsia 48:2086-2092.

Reuber M. (2008) Psychogenic nonepileptic seizures: answers and questions. Epilepsy Behav 12:622-635.

Reuber M, Fernandez G, Bauer J, Helmstaedter C, Elger CE. (2002) Diagnostic delay in psychogenic nonepileptic seizures. Neurology 58:493-495.

Selwa LM, Geyer J, Nikakhtar N, Brown MB, Schuh LA, Drury I. (2000) Nonepileptic seizure outcome varies by type of spell and duration of illness. Epilepsia 41:1330-1334.

Seneviratne U, Reutens D, D'souza W. (2010) Stereotypy of psychogenic nonepileptic seizures: insights from video-EEG monitoring. Epilepsia 51:1159-1168.

Walczak TS, Bogolioubov A. (1996) Weeping during psychogenic nonepileptic seizures. Epilepsia 37:208-210. 